\title{
Formation of Uniform Germanium Islands on Silicon Substrate Using Nickel as Catalyst by Thermal Evaporation Method
}

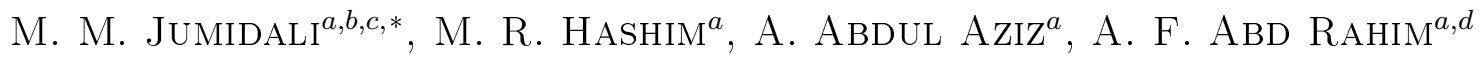 \\ ${ }^{a}$ Nano-Optoelectronics Research Laboratory, School of Physics, \\ Universiti Sains Malaysia 11800, Pulau-Pinang, Malaysia \\ ${ }^{b}$ Faculty of Applied Sciences, Universiti Teknologi MARA Malaysia, 40450 Malaysia \\ ${ }^{c}$ Department of Applied Sciences, Universiti Teknologi \\ MARA Pulau Pinang, 13500 Pulau Pinang, Malaysia \\ ${ }^{d}$ Faculty of Electrical Engineering, Universiti Teknologi \\ MARA Pulau Pinang, 13500 Pulau Pinang, Malaysia
}

\begin{abstract}
Uniform germanium islands (GIs) were grown on $\mathrm{Si}(100)$ using a nickel layer as catalyst through the physical vapor deposition of germanium $(\mathrm{Ge})$ powder at $1000^{\circ} \mathrm{C}$ at different deposition times. Prior to the deposition of Ge layer, nickel $(\mathrm{Ni})$ catalyst was deposited on silicon substrates via radio frequency magnetron sputtering technique. Scanning electron microscopy results showed that the increase in deposition time resulted in a variation in surface morphology. Energy dispersive X-ray spectrometer analysis found that the GI samples composed of Ni element indicating the role of $\mathrm{Ni}$ in uniform $\mathrm{Ge}$ islands formation. The X-ray diffraction pattern spectra revealed that the GIs exhibited a Ge cubic structure and the intensity of Ge peaks varies with deposition time. In-plane strain indicated that the strain caused by the substrate is tensile and changed to compressive strain at the longer deposition time. The Raman spectra exhibited a red shift in the Ge-Ge peak, compared with the bulk Ge, as a result of compressive strain of the GIs. Fourier transform infrared spectrum analysis also indicated that the optical band gap $E g$ values of GIs can be varied by deposition time.
\end{abstract}

DOI: 10.12693 /APhysPolA.127.1068

PACS: 81.05.Ea, 81.10.Bk, 81.15.-z

\section{Introduction}

The formation mechanisms and properties of germanium islands (GIs) on Si structures are of great interest for use in new optoelectronic devices. Several methods are employed to fabricate GIs with different sizes, such as chemical vapor deposition $[1,2]$, radio frequency magnetron sputtering [3, 4], molecular beam epitaxy [5-7], and thermal evaporation [8]. However, establishing a method to achieve sufficiently uniform island sizes with regular spatial distribution remains a critical issue. Substantial research focused on the size distribution of the islands because such islands are an important aspect in practical application [9]. The conventional way to control island formation (size, shape and density) is to vary growth conditions by altering substrate temperature and molecular flux [9].

Metals modify nucleation and growth phenomena normally used to tune the characteristics of epitaxial islands. Nickel (Ni) is a material that has promising use as a metal catalyst. For instance, Tuan et al. [10] and Hsu et al. [11] successfully synthesized $\mathrm{Ge}$ and $\mathrm{Si}$ nanowires using $\mathrm{Ni}$

\footnotetext{
* corresponding author; e-mail: mohdmuza433@ppinang.uitm.edu.my
}

catalysts. Recently, Kolahdouz et al. [12] used $\mathrm{Ni}$ as a metal catalyst to form islands based on substrate engineering to control the diameter of carbon multi-walled nano-tubes. The use of $\mathrm{Ni}$ as a catalyst for GI growth via thermal evaporation is very promising because, thermodynamically, $\mathrm{Ni}$ is easy to agglomerate with $\mathrm{Ge}$. The $\mathrm{Ni} / \mathrm{Ge}$ alloy has a low eutectic temperature at $762^{\circ} \mathrm{C}$. This character allows the easy formation of $\mathrm{Ni} / \mathrm{Ge}$ alloy liquid droplets on a Si substrate and serves as a trap of growth species for GI formation. In this paper, we report on a fabrication of GI on an n-Si substrate grown via simple thermal evaporation method using a $\mathrm{Ni}$ epitaxial layer as catalyst. The roles of $\mathrm{Ni}$ in GI formation on Si (100) necessitate further study because no such report is available in the literature. Significant different GI behaviors are observed when deposition time varies. The structural and optical properties of the formed islands are characterized and investigated.

\section{Experimental procedures}

The germanium islands (GIs) were synthesized in a horizontal tube furnace system by the simple thermal evaporation method in the presence of nickel $(\mathrm{Ni})$ metal layer on the silicon ( $\mathrm{Si}$ ) substrate as the catalyst. The Ni-coated Si substrates (thickness $\sim 250 \mathrm{~nm}$ ) were prepared by A500 Edwards RF magnetron sputtering system. The Ni-coated substrates were placed upside down 
on top of the alumina boat to act as substrate for GIs growth. The $99.999 \%$ of Ge powder act as starting materials were then heated up to $1000^{\circ} \mathrm{C}$ under a Nitrogen $\left(\mathrm{N}_{2}\right)$ atmosphere for different hours. To study the heating time effect, the samples were heated to a particular time in a flow of $\mathrm{N}_{2}$ gas. After deposition process, the samples were drawn out and cooled to room temperature for analysis. The as-grown products were structurally characterized by a X-ray diffractometer (PAN analytical X'Pert PRO diffractometer with $\mathrm{Cu}-\mathrm{K}_{\alpha}$ radiation). Scanning electron microscopy (JEOL JSM-6460LV with energy dispersive X-ray spectroscopy, EDX) was used to analyze the morphology of the products. Room temperature Raman spectrum was taken with a Raman spectrometer (Horiba Jobin Yvon HR800), with Ar+ as excition source operating at a wavelength of $514.55 \mathrm{~nm}$. The FTIR spectra were collected by a Perkin Elmer Fourier Transform Infrared Spectrometer for characterizing the optical properties of GIs.

\section{Results and discussion}

Figure 1a to 1d show the scanning electron microscopy (SEM) images of synthesized GIs on a Ni-coated Si substrate for different growth times (from $1 \mathrm{~h}$ to $3 \mathrm{~h}$ ). The images revealed that different GI shapes are distributed randomly on the Si surface as the deposition time increases. Figure 1a show the images of sample growth for $1 \mathrm{~h}$, which consists of small- and medium-sized sphere GIs. All the islands were grown randomly, and some of a $\mathrm{Ni}$ cluster remained on the $\mathrm{Si}$ substrate. As the growth time increased to $2 \mathrm{~h}$, GI morphology on the Si substrate changed to the bigger size of spherical shaped GIs without any remaining Ni cluster on the Si substrate (Fig. 1b). The increase on size of GIs affirmed that the GIs were coarsening (i.e., the movement of nucleated GIs from smaller to larger islands results in increased GI size). Increasing the heating time to $3 \mathrm{~h}$ (Fig. 1c) resulted in the formation of irregular shape of GIs on the substrate. Higher magnification image (Fig. 1d) confirmed that the irregular shape of GIs due to formation of $\mathrm{SiO}_{2}$ around the islands (the samples have mushroom-like feature). Energy-dispersive X-ray spectroscopy (EDX) analysis was performed on the particular islands to study the distribution of material compounds on the island. The EDX images shown in the inset of Fig. 1a, $1 \mathrm{~b}$ and $1 \mathrm{c}$ indicate that all the GIs consisted primarily of Ge and $\mathrm{Ni}$ elements, as well as small amounts of $\mathrm{Si}$ and $\mathrm{O}$ elements in sample 1 and 2 hour. Sample for 3 hour shows the highest $\mathrm{Si}$ and $\mathrm{O}$ elements suggested the GIs were contaminated by $\mathrm{SiO}_{2}$. Ni content was clearly preserved as the deposition time increased, which also shows the role of $\mathrm{Ni}$ in GIs formation.

Figures $2 \mathrm{a}$ to $2 \mathrm{c}$ illustrate the XRD patterns of the GI film prepared at different heating times for 1, 2, and $3 \mathrm{~h}$, respectively. The diffraction peaks observed in the produced GIs film mainly corresponds to a cubic-type Ge structure with preferred orientations at the (111),

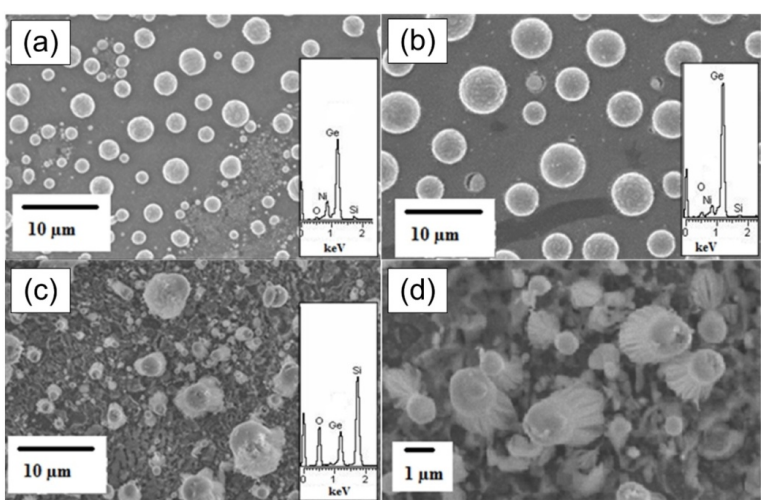

Fig. 1. The SEM images and inset EDX spectra of GIs grown on Si substrate for (a) 1 hour, (b) 2 hours, (c) 3 hours deposition times respectively and (d) high magnification of SEM image of 3 hours deposition time.

(220), and (311) planes. The intensity of the Ge peaks increase as deposition time increase from one to two hours deposition time and rapidly decreased at longer deposition times ( 3 hour). The decrease of the Ge intensity peaks suggested that the GIs were covered by $\mathrm{SiO}_{2}$. The nanometer-scale amorphous $\mathrm{Si}$ was reported to be vaporized above $1000^{\circ} \mathrm{C}$ [13]. Therefore, at the longer heating times the amorphous $\mathrm{Si}$ vapor from Si substrate will possibly diffuse in GIs structures and with easily presence of oxygen at high temperature will allow the formation of $\mathrm{SiO}_{2}$. The existence of the $\mathrm{Ni} / \mathrm{Ge}$ alloy on each sample shows the role of $\mathrm{Ni}$ in the formation of GI structures. Table I summarizes the full width at half

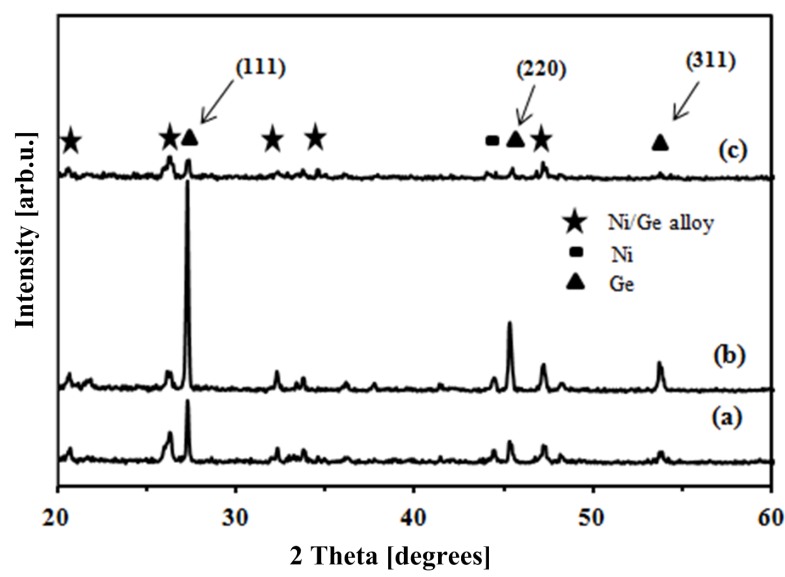

Fig. 2. XRD patterns of the GI film prepared at different heating times for (a) 1 , (b) 2, and (c) 3 hours, respectively.

maximum (FWHM) of the (111) plane, crystallite size $\left(D_{P}\right)$, lattice constant, and strain for the GI films prepared at different deposition times. With an increase in deposition time, the FWHM becomes narrower; hence, the crystallite size increases. The increasing crystallite size can be due to the increasing number of Ge particles 
collected during the longer deposition time. The lattice constant results also shown increases suggested that the unit cell is elongated along the axis and the compressive forces act in the plane of the film. The lattice constant can be further utilized to evaluate the average uniform strain in the lattice along the a-axis. The positive value of the in-plane strain $\varepsilon_{a}$ for the 1 hour and two hour deposition times indicates that the strain caused by the substrate is tensile. The tensile strain values increased when heating time was increased are in good agreement with the results reported by Hartmann et al. [14]. The negative value of the in-plane strain $\varepsilon_{a}$ for the longer deposition time ( 3 hour) shows that the strain caused by the substrate is compressive. Figure 3 shows the Raman

XRD data of GIs growth

TABLE I

\begin{tabular}{c|c|c|c|c|c}
\hline \hline $\begin{array}{c}\text { Deposition } \\
\text { Time } \\
\text { [Hour] }\end{array}$ & $2 \theta$ & $\begin{array}{c}\text { FWHM } \\
{[\mathrm{nm}]}\end{array}$ & $\begin{array}{c}\mathrm{D}_{P} \\
{[\mathrm{~nm}]}\end{array}$ & $\begin{array}{c}\text { Lattice } \\
\text { constant } \\
\mathrm{a}[\AA]\end{array}$ & $\begin{array}{c}\text { Strain } \\
\left(\varepsilon_{a}\right) \\
\times 10^{-3}\end{array}$ \\
\hline 1 & 27.2851 & 0.2952 & 27.69 & 5.66133 & +0.234982 \\
2 & 27.2735 & 0.1968 & 41.54 & 5.66370 & +0.653710 \\
3 & 27.3025 & 0.1968 & 41.54 & 5.65783 & -0.383392
\end{tabular}

spectra of the three samples grown on Si at different deposition times. With increased deposition time, the samples show a decrease in peak intensity and an increase in FWHM, demonstrating that the quality of the GIs is reduced when heating time is increased.

The peaks around $300 \mathrm{~cm}^{-1}$ are attributed to the optical phonon contribution of the Ge-Ge stretching mode [15]. The red-shifted trend for sample grown compared to that of the bulk $\mathrm{Ge}\left(300 \mathrm{~cm}^{-1}\right)[16]$ is compatible with the previous XRD result, which suggests that the sample experienced tensile strain for 1 and 2 hour deposition and compressive strain for 3 hour heating times. Another low and broad hump peak centered at about $446.0 \mathrm{~cm}^{-1}$. There are no reports of the Raman peak for $\mathrm{Ni}-\mathrm{Ge}$ alloys in the literature and according to our XRD spectra, the peak at $446.0 \mathrm{~cm}^{-1}$ is suggested attribute to the $\mathrm{Ni}-\mathrm{Ge}$ phase. The absence of $\mathrm{Si} / \mathrm{Ge}$ vibration peaks about $400 \mathrm{~cm}^{-1}$ corresponds to the $\mathrm{Si} / \mathrm{Ge}$ alloy mode [17] implies there is no intermixing of $\mathrm{Si}$ from the substrate with GIs. The higher peak at $520 \mathrm{~cm}^{-1}$ is attributed to the optical phonon mode of the Si substrate [18]. The optical band gap $(E g)$ for GI films were determined by analyzing the optical data, with the expression of the optical absorbance $\alpha$ and the incident photon energy $h \nu$, using the Tauc equation [19]. The band gaps were determined by plotting $(\alpha h v)^{3 / 2}$ versus $h \nu$ (Fig. 4$)$. The optical results (Fig. 4) have shown that $E g$ was found to decrease as heating time increased. This finding is in good agreement with the results reported [20]. The lower values of the energy band gap are attributable to the well known close relationship between the band gap and crystallites size. In another related observation, the $E g$ red shift can be due to an increase in crystal intrinsic defects as a result of impurities that come from the formation of $\mathrm{SiO}_{2}$ in the GIs structure due to longer heating time.

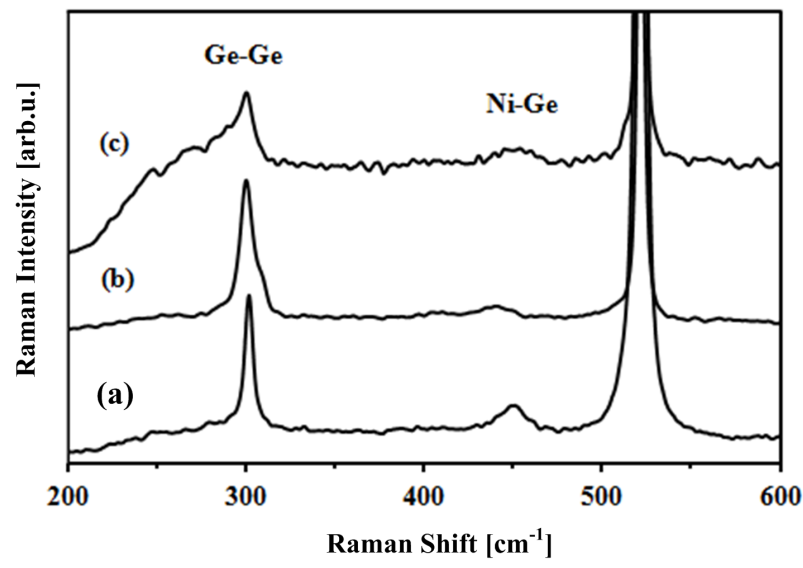

Fig. 3. Raman spectra of the GI film prepared at different heating times for (a) 1, (b) 2, and (c) 3 hours, respectively.

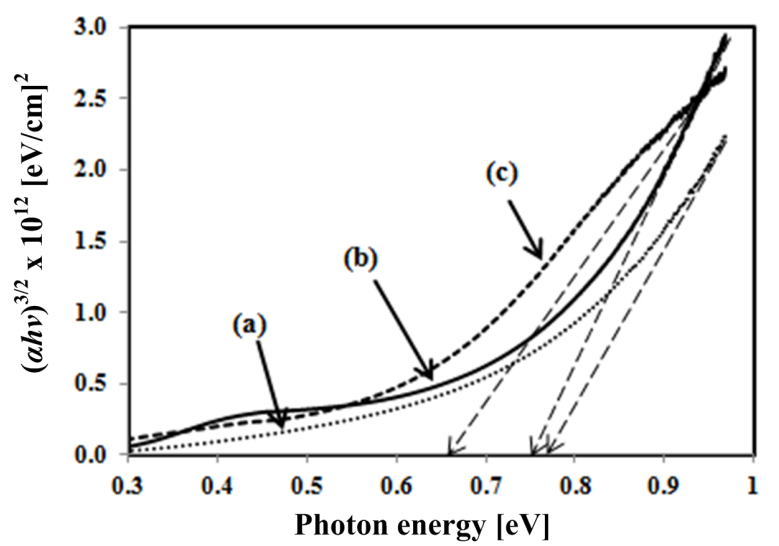

Fig. 4. $(\alpha h \nu)^{3 / 2}$ vs $h v$ plot of GIs with different deposition time: (a) $1 \mathrm{~h}$, (b) $2 \mathrm{~h}$, and (c) 3 hours.

\section{Conclusions}

In summary, the morphological, structural, and optical properties of GIs synthesized through simple thermal evaporation using a $\mathrm{Ni}$ catalyst were investigated. SEM images showed that the morphology of GI structures changes with different deposition times. The XRD measurements revealed that GIs have good crystal quality of crystalline Ge cubic phase and a small amount of crystalline $\mathrm{Ni} / \mathrm{Ge}$ alloy. Room-temperature Raman scattering indicated that the samples were in the $\mathrm{Ge}-\mathrm{Ge}$ stretching mode and red-shifted as the longer deposition time. The energy band gap decreased as the deposition time increased because of the increase in particle size in the GIs.

\section{Acknowledgments}

The authors would like to thank Universiti Sains Malaysia, Penang for the provision of financial support under USM Incentive Grant 1001/PFIZIK/822078 
and USM-RU-PRGS Grant 1001/PFIZIK/843006. Also the financial support of the Universiti Teknologi MARA through UiTM/KPT scholarship is gratefully acknowledged.

\section{References}

[1] G. Capellini, L. Di Gaspare, F. Evangelisti, E. Palange, Applied Physics Letters 70, 493 (1997).

[2] M. Borgström, V. Zela, W. Seifert, Nanotechnology 14, 264 (2003).

[3] K. Das, M. Goswami, A. Dhar, B. Mathur, S. Ray, Nanotechnology 18, 175301 (2007).

[4] A. Samavati, Z. Othaman, S. Ghoshal, M. Dousti, R. Amjad, Chinese Physics Letters 29, 118101 (2012).

[5] K.-F. Wang, Y. Zhang, W. Zhang, Applied Surface Science 258, 1935 (2012).

[6] I. Goldfarb, L. Banks-Sills, R. Eliasi, Applied Physics Letters 85, (2004).

[7] T. Merdzhanova, S. Kiravittaya, A. Rastelli, M. Stoffel, U. Denker, O. Schmidt, Physical Review Letters 96, 226103 (2006).

[8] I. Kovačević, B. Pivac, P. Dubček, H. Zorc, N. Radić, S. Bernstorff, M. Campione, A. Sassella, Applied Surface Science 253, 3034 (2007).

[9] A.V. Dvurechenskii, J.V. Smagina, R. Groetzschel, V.A. Zinovyev, V.A. Armbrister, P.L. Novikov, S.A. Teys, A.K. Gutakovskii, Surface and Coatings Technology 196, 25 (2005).
[10] H.-Y. Tuan, D.C. Lee, T. Hanrath, B.A. Korgel, Chemistry of materials 17, 5705 (2005).

[11] J.-F. Hsu, B.-R. Huang, Thin Solid Films 514, 20 (2006).

[12] Z. Kolahdouz, M. Kolahdouz, H. Ghanbari, S. Mohajerzadeh, S. Naureen, H.H. Radamson, Materials Science and Engineering: B 177, 1542 (2012).

[13] J.-P. Borel, Surface Science 106, 1 (1981).

[14] J. Hartmann et al., Journal of Applied Physics 95, 5905 (2004).

[15] G. Kartopu, S.C. Bayliss, V.A. Karavanskii, R.J. Curry, R. Turan, A.V. Sapelkin, Journal of Luminescence 101, 275 (2003).

[16] D.R. dos Santos, I.L. Torriani, Solid State Communications 85, 307 (1993).

[17] M. Fujii, S. Hayashi, K. Yamamoto, Applied Physics Letters 57, 2692 (1990).

[18] R. Al Asmar, J.P. Atanas, M. Ajaka, Y. Zaatar, G. Ferblantier, J.L. Sauvajol, J. Jabbour, S. Juillaget, A. Foucaran, Journal of Crystal Growth 279, 394 (2005).

[19] J. Tauc, in: Amorphous and Liquid Semiconductors 4: Plenum Press, New York USA, 1974.

[20] N.V. Vostokov, Z.F. Krasil'nik, D.N. Lobanov, A.V. Novikov, M.V. Shaleev, A.N. Yablonskii, Phys. Solid State 46, 60 (2004). 